УДК 621.574 .4

\title{
СРАВНИТЕЛЬНЫЙ АНАЛИЗ СПЕКТРАЛЬНЫХ ХАРАКТЕРИСТИК СИГНАЛОВ ПОДПОВЕРХНОСТНЫХ РЛС ДЛЯ ОЦЕНКИ ДОРОЖНОГО ПОКРЫТИЯ
}

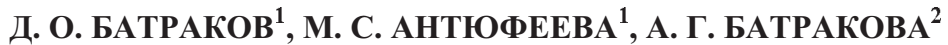 \\ ${ }^{1}$ Харьковский национальный университет имени В. Н. Каразина \\ Украина, Харьков, 61077, пл. Свободы, 4 \\ ${ }^{2}$ Харьковский начиональный автомобильно-дорожный университет \\ Украина, Харьков, 61002, ул. Ярослава Мудрого, 25
}

\begin{abstract}
Аннотация. В работе представлены результаты экспериментов по использованию сверхширокополосных РЛС подповерхностного зондирования (СШП РЛСПЗ) с трехантенными модулями для систем неразрушающего контроля. Использованные модули имеют различные значения центральных частот зондирующих СШП импульсов и различные конфигурации антенн. Цель проведенных экспериментов заключалась в отыскании наилучших значений параметров чувствительности. Другая задача состояла в оптимизации метода обработки сигналов, отраженных от плоскослоистых сред. Проведенный анализ полученных результатов показал пути улучшения качества неразрушающего контроля дорожного покрытия и других строительных структур при помощи СШП РЛСПЗ. Предлагаемый подход основан на ранее предложенном алгоритме поэтапного определения толщины слоев дорожного покрытия и использовании относительной диэлектрической проницаемости.
\end{abstract}

Ключевые слова: неразрушающий контроль; сверхширокополосный сигнал; СШП сигнал; подповерхностная РЛС; георадар; дорожное покрытие

\section{1. ВВЕДЕНИЕ}

Распространенные задачи использования РЛС подповерхностного зондирования (георадар, РЛСПЗ) для неразрушающего контроля различных строительных конструкций и объектов транспортной инфраструктуры, в первую очередь дорог, рассмотрены во многих монографиях $[1,2]$. Проблемы применения математических моделей для интерпретации массивов данных также рассмотрены в [4, 5], а результаты использования РЛСПЗ для решения практических задач обсуждались в технических отчетах $[6,7]$.

Следует отметить, что улучшение средств зондирования (РЛСПЗ) и рост возможностей компьютеров, не только способствуют про- грессу в решении практических задач, но также открывают новые возможности для совершенствования теоретических методов и алгоритмов, предложенных ранее.

Например, увеличение центральной частоты РЛСПЗ позволило увеличить разрешающую способность при определении толщины слоев плоскослоистой среды $[8,9]$. В дорожном хозяйстве это позволяет подойти к такой практически важной проблеме как обнаружение потери межслойного сцепления $[10,11]$. В данном случае подразумевается, что задача надежного обнаружения потери межслойного сцепления имеет собственные особенности и является весьма сложной. 
На данный момент возможно вести речь только о некоторых особых ситуациях (присутствие воздушного зазора или присутствие воды между слоями), позволяющих с высокой вероятностью обнаружить потерю межслойного сцепления. В других случаях, новые возможности контроля и координации радаров, расположенных на одной движущейся лаборатории, позволяют обеспечить одновременное использование нескольких устройств для получения более полных массивов данных [8].

В свою очередь, эти новые возможности потребовали разработки новых методов и вычислительных алгоритмов для обработки и интерпретации результирующих массивов данных. Среди этих методов следует выделить методы, ориентированные на визуальную обработку радарограмм [12], и методы, основанные на анализе временного распределения сигналов, которые отражаются исследуемой средой [2, 13-15].

Подход [2] теоретически позволяет реализовать поэтапный алгоритм для последовательного определения значений диэлектрической постоянной слоев и их толщин. Однако опыт реального использования такой антенной системы с электромагнитной развязкой для СШП импульсной РЛСПЗ для мониторинга дорожных покрытий показывает, что измерения фактически выполняются в ближней или промежуточной зоне антенн. Более того, некоторые верхние слои дорожного покрытия лежат в ближней зоне, а нижние слои находятся в промежуточной зоне. Это приводит к отклонению поведения дифрагированного поля от модели рассеяния плоской волны, хотя такая модель (формулы Френеля) используется многими авторами $[1,16,17]$.

Одним из предложенных методов для решения этой задачи является предварительная калибровка и введение необходимого коэффициента, который учитывает нелинейное поведение поля в зависимости как от расстояния до структурных границ, так и от диэлектрической проницаемости слоистых материалов [2, 18, 19].

Следует подчеркнуть, что задачи дефектометрии непосредственно связаны с задачей измерения толщины. Дело в том, что если задачи обнаружения наличия дефектов могут быть решены оператором с помощью визуальной обработки радарограмм, то задачи определения основных параметров дефектов, таких как трещины (дефектометрия), могут быть решены только на основе комбинированных алгоритмов.

Такие алгоритмы включают одну часть, обеспечивающую определение толщины слоев, и другую часть, для определения параметров дефекта, т.е. трещин. В результате, становится возможным не только обнаружить дефект, но также идентифицировать его, т. е., определить, трещина ли это, или заполнение технологического шва, а также выяснить его положение. В случае трещины, под термином положение трещины подразумевается определение ее направления, ширины зазора, материал заполнения и глубина [20].

Очевидно, что решение этих задач невозможно без тщательного анализа особенностей антенных систем различных конструкций с различными спектральными параметрами, а также без разработки эффективных моделей для обработки и интерпретации данных.

Практическая задача состоит в нахождении наиболее эффективного технического решения для задачи измерения толщины слоев плоскослоистых сред (например, дорожных покрытий). Таким образом, основная цель этой работы состоит в изучении возможностей спектральной обработки СШП импульсов с различными центральными частотами и при различных принципах регистрации принятых сигналов. Другая цель состоит в повышении надежности и точности определения толщины слоев. 


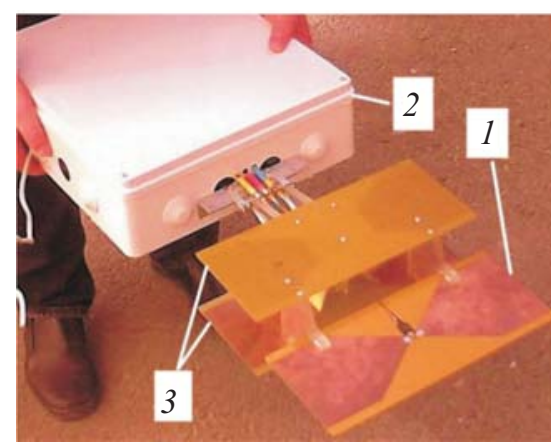

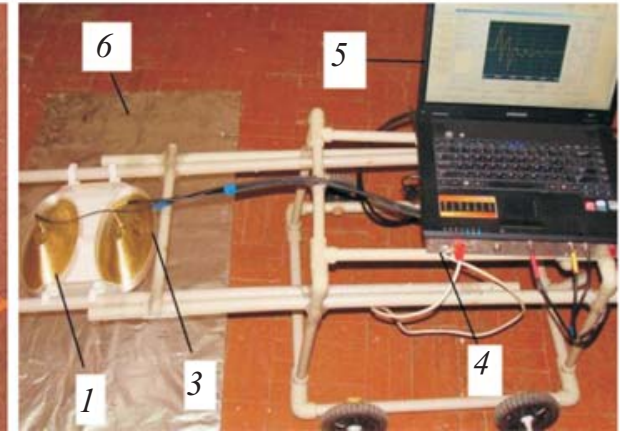

б

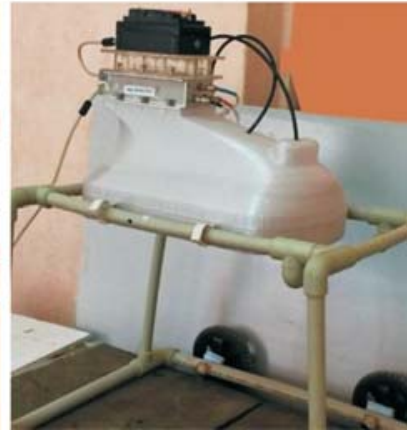

B

Рис. 1. Георадары ТРФ-3 (a), Одяг-5 (б) и Одяг-4 (в). Здесь: 1 - передающая антенна,

2 - модуль дифференциальной принимающей антенны, 3 - принимающая антенна, 4 - блок электроники, 5 - ноутбук, 6 - металлический лист.

\section{2. ФОРМУЛИРОВКА И РЕШЕНИЕ ЗАДАЧИ}

С точки зрения математики, задача состоит в изучении спектральных характеристик реальных сигналов как для антенной системы с электромагнитной развязкой, так и для новых рупорных антенн (рис. 1б) для импульсных СШП РЛСПЗ.

Значимость этой задачи определяется тем фактом, что в процессе процедуры предварительной калибровки для определения амплитуды зондирующего сигнала вначале регистрируется сигнал помехи от непосредственной связи между передающей и приемной антеннами. Затем амплитуда входного сигнала, представляющая собой зафиксированное отражение от металлического листа, лежащего на поверхности проверяемого дорожного покрытия, фиксируется непосредственно перед измерениями. В результате, спектр сигнала прямой связи, и спектр сигнала, отраженного от металлического листа, имеют существенные отличия от модельных сигналов, синтезированных на компьютере [21].

В связи с этим необходимо уяснить природу и причины отклонений спектров на первом этапе, и продолжить поиск путей для решения этой проблемы и разработки вычислительных алгоритмов для обработки данных. В качестве модели исследуемой среды для обработки отраженных сигналов использована модель плоскослоистой среды на однородной полубесконечной подложке без включений $[2,21]$. Другими словами, предполагается, что только неоднородности, вызывающие отражение электромагнитных волн, являются границами между слоями.

\section{3. УСЛОВИЯ ПРОВЕДЕНИЯ ЭКСПЕРИМЕНТА}

Для получения начальных данных зондирования, которые в дальнейшем служат основой для численного моделирования, используются импульсные СШП сигналы от трех георадаров, имеющих различные центральные частоты. Под центральной частотой, как и ранее [21], понимается не частота с максимальной спектральной амплитудой, а центральная частота спектра, т.е. мера центральной частоты между верхней и нижней граничными частотами.

Такой выбор сделан авторами, поскольку имеется несколько георадаров (рис. 1) с различными значениями центральной частоты спектра импульсного сигнала для контроля дорожного покрытия.

Центральная частота РЛС подповерхностного зондирования ТРФ-3 (рис. 1) является самой низкой и составляет $f_{\mathrm{c}}^{\mathrm{T} 3}=500$ МГц. Два других георадара Одяг-5 (рис. 1б) и Одяг-4 (рис. 1в) имеют более высокие значения цен- 

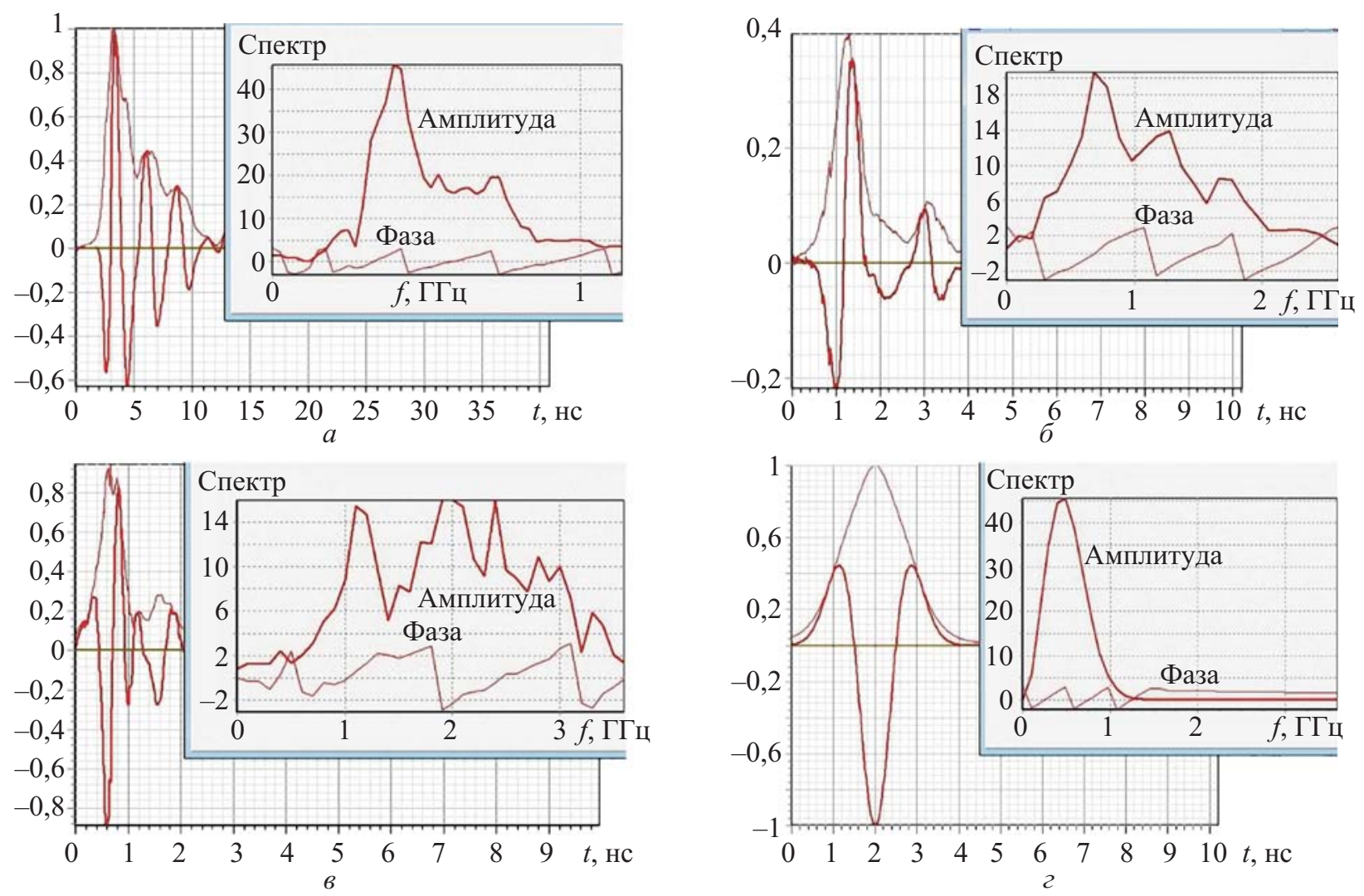

Рис. 2. Временное распределение зондирующего сигнала и его спектр для ТРФ-3 (а), Одяг-5 (б) и Одяг-4 (в) и компьютерное моделирование временного распределения импульсного сигнала и его спектра (г) Толстая линия — спектральная амплитуда, тонкая линия - фаза.

тральной частоты, составляющие примерно $f_{\mathrm{c}}^{\mathrm{O} 5}=1050$ МГц и $f_{\mathrm{c}}^{\mathrm{O} 4}=1600$ МГц. Формы зондирующих сигналов этих георадаров и их спектральные характеристики показаны на рис. 2. Процедура получения этих данных подробно описана в $[2,18,19]$.

\section{4. РЕЗУЛЬТАТЫ ЭКСПЕРИМЕНТА И ОБСУЖДЕНИЕ}

На рис. 2 показаны распределения сигналов во времени (слева) и рассчитанные распределения спектральных амплитуд и фаз соответствующих сигналов (справа). Временная зависимость исследуемого сигнала отображается в окне «Анализ сигнала», а частотные зависимости спектральных амплитуд и фаз этого сигнала отображаются в окне справа вверху.
Эти зависимости полностью определяют исследуемый сигнал, который можно точно восстановить с помощью обратного преобразования Фурье.

Отметим, что форма спектральных кривых реальных антенных систем георадаров отличается от формы аналитических сигналов, которые являются второй производной от гауссова распределения, которая в научной литературе известна под названием «сомбреро». Это обусловлено тем фактом, что импульс, отраженный от листа металла, фиксируется как зондирующий сигнал в соответствии с предварительным измерением процедуры сигнала прямой связи, и последующей калибровки для измерения отражения от металлического листа. 
Этот лист металла помещается на поверхность дорожного покрытия. В этом случае, высота антенного модуля над металлом (поверхностью дорожного покрытия) составляет 25-40 см. Из теории электромагнитного поля и теории антенн следует, что данное измерение осуществляется в промежуточной зоне, а в некоторых случаях - в ближней зоне. При этом металлический лист имеет ограниченные размеры и прямоугольную форму, а материал обладает конечной проводимостью. Возможны отражения от границ (ребер) листа и его углов, возможны также повторные отражения сигналов от поверхности листа, которые однажды отразились от листа и затем отразились от металлической антенны, после чего снова упали на поверхность листа. К сожалению, невозможно полностью исключить влияние этих факторов и достичь распределения, близкого к теоретическому спектральному распределению. Теоретический вид временного распределения идеального импульса и его спектральное распределение показаны на рис. 22 .

Одним из возможных путей решения проблемы несоответствия сигналов (отличие их характеристик от эталона) является обработка сигнальных спектров. Ранее в [22-26] предложено несколько алгоритмов для реализации этого подхода. В основном они сосредоточены на регистрации частотных зависимостей коэффициентов отражения плоских волн на зондируемой среде. Этот подход является эффективным при решении обратных задач по восстановлению непрерывного плавно изменяющегося распределения диэлектрической проницаемости.

Для решения задач определения толщины слоев ранее предложены методы для обработки импульсных сигналов, которые позволяют определить толщину и значение диэлектрической постоянной среды, состоящей из нескольких слоев. Эти слои могут иметь как значительную $[2,13]$, так и малую толщину $[18,19]$.
Для увеличения разрешающей способности по толщине, стандартный метод предполагает использование георадара с высокой центральной частотой. Однако при увеличении частоты, ослабление электромагнитных волн также возрастает, что ведет к ограничению глубины зондирования.

В процессе поиска оптимальных значений центральной частоты георадара использована обработка данных с помощью программы GeoVizy $[18,19]$. Цель экспериментальных исследований состояла в анализе результатов, полученных при использовании импульсных сигналов с различными центральными частотами, и рассмотрении возможностей улучшения качества обработки данных.

\section{5. ОБРАБОТКА РЕЗУЛЬТАТОВ}

Для обработки данных зондирования ранее предложено два интегральных преобразования. Первое - преобразование Гильберта для сигнала, описываемого функцией $x(t)$ [2, 18]:

$$
H[x(t)]=\frac{1}{\pi} \int_{-\infty}^{\infty} \frac{x(\tau)}{t-\tau} \mathrm{d} \tau .
$$

При использовании преобразования Гильберта аналитический сигнал строится как положительная функция $S(t)$ :

$$
S(t)=|h(t)|=\sqrt{x^{2}(t)+\tilde{x}^{2}}
$$

и затем на оси времени определяется расстояние между максимумами этой функции.

Второе преобразование предложено в [29, 30], и предполагает двойное интегрирование временной зависимости сигнала с переменным верхним пределом. Благодаря хорошо известному свойству интеграла с переменным верхним пределом (его производная относительно верхнего предела равна подынтегральному выражению и вычисляется для этого предела) вместо вычисления двойного интеграла и по- 


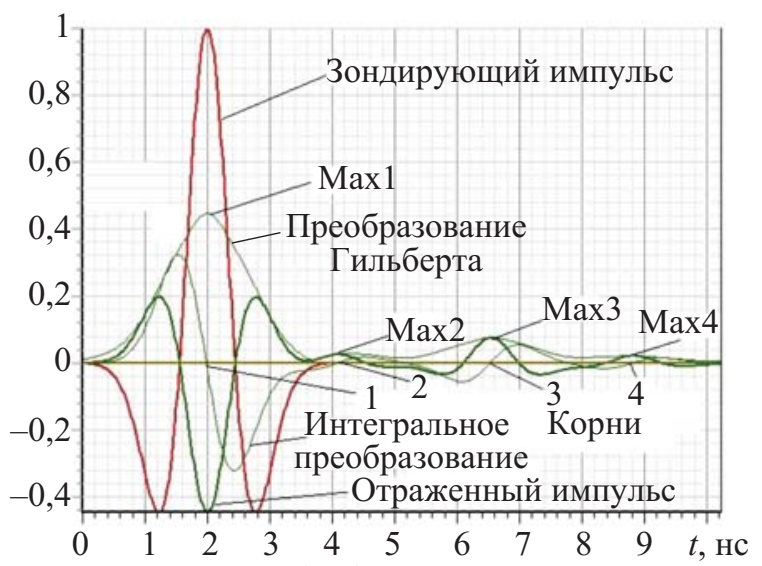

Рис. 3. Пример обработки моделированного сигнала.

следующего определения экстремумов (через нули производной), достаточно сразу вычислить нули производной, как корни обычного интеграла с переменным верхним пределом с помощью сигнала, зафиксированного в точке приемной антенны $e\left(t, \mathbf{r}_{0}\right)$ :

$$
S\left(t, \mathbf{r}_{0}\right)=\int_{0}^{t} e\left(\tau, \mathbf{r}_{0}\right) \mathrm{d} \tau .
$$

В этом случае, задача сводится к нахождению локальных максимумов функции $S(t)$ (2) и нулей функции $S\left(t, \mathbf{r}_{0}\right)(3)$. Для сигналов, синтезированных с помощью компьютерных программ, эти точки всегда совпадают (рис. 3). На рис. 3 и 4 показаны два импульсных сигнала. Зондирующий импульс (рис. 3) моделируется с помощью идеального СШП импульса, который рассчитывается как вторая производная гауссовой функции:

$$
S_{g}(t)=A\left(N_{s}\left(t-t_{i}\right)^{2}-1\right) \mathrm{e}^{-\left(N_{s} \frac{\left(t-t_{i}\right)^{2}}{2}\right)},
$$

где $A=1 / N_{s}$ - амплитуда сигнала, $t_{i}$ - время сдвига центра импульса $\left(t_{i}=2 \mathrm{Hc}\right), N_{s}$ - параметр, характеризующий крутизну фронта импульса $\left(N_{s}=5\right)$.

На рис. 4 показан фрагмент сигнала реального георадара Одяг-4. Этот рисунок получен

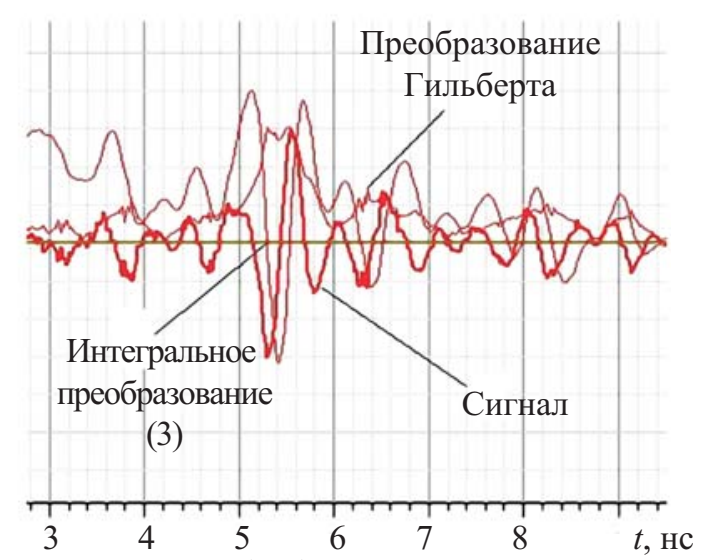

Рис. 4. Пример обработки сигнала георадара Одяг-4.

при обработке реального сигнала РЛСПЗ Одяг-4, отраженного от двухслойной структуры. Первый слой представляет собой сухой песок толщиной 15 см, второй слой - влажная глина толщиной 19 см. Наличие другого сигнала $(t=9,3$ нс) также представляет интерес. Скорее всего этот сигнал вызван повторным многократным отражением между наружной поверхностью и первой границей структуры. Однако этот вопрос требует дополнительного исследования.

С одной стороны, рис. 4 демонстрирует преимущества совместного использования двух преобразований, описываемых выражениями (2) и (3), для увеличения надежности обработки данных, а с другой стороны, он указывает на сложность физических процессов, сопровождающих взаимодействие импульсных сигналов антенных модулей реального георадара в случае плоскослоистых сред.

\section{6. МОДЕЛИ И ПРЕДЛАГАЕМЫЙ МЕТОД}

Для решения задачи оптимизации создано несколько моделей исследуемой среды. Одна из этих моделей включает три слоя различной толщины. Эти слои расположены на полубесконечной подложке. Два верхних слоя имеют сравнительно близкие значения диэлектрической проницаемости, равные 6,8 и 6,0 , соответственно. Значения других пара- 


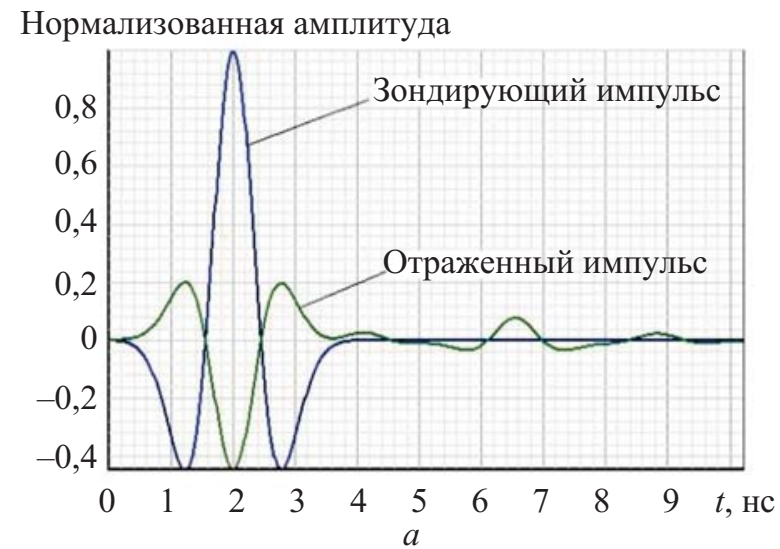

Нормализованная амплитуда

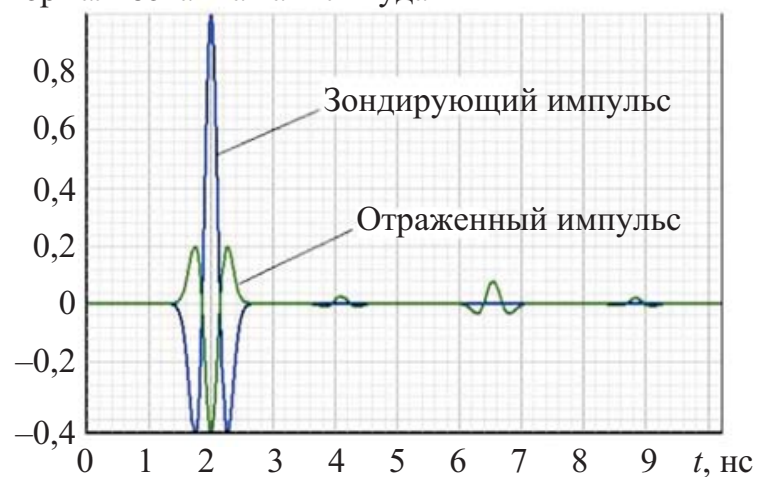

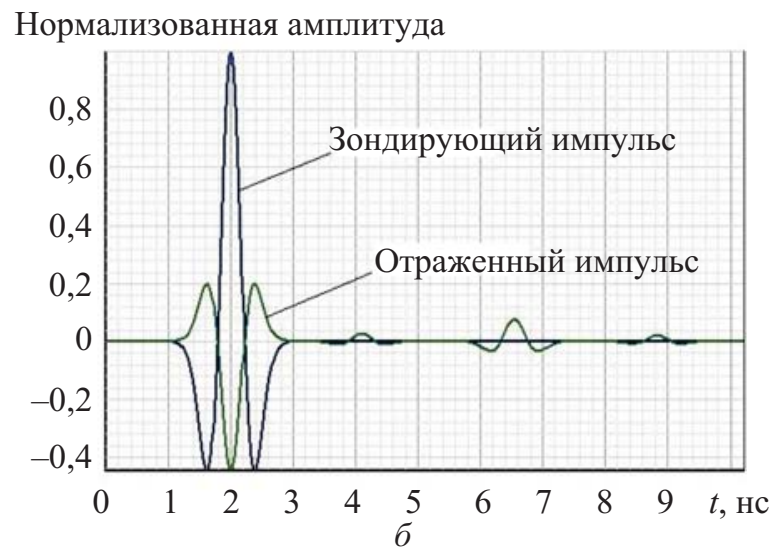

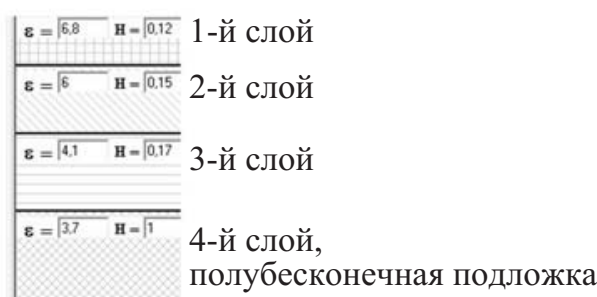

Рис. 5. Моделирование зондирования импульсом с частотой $f_{c}=500(a), 1050(б), 1600$ МГц (в).
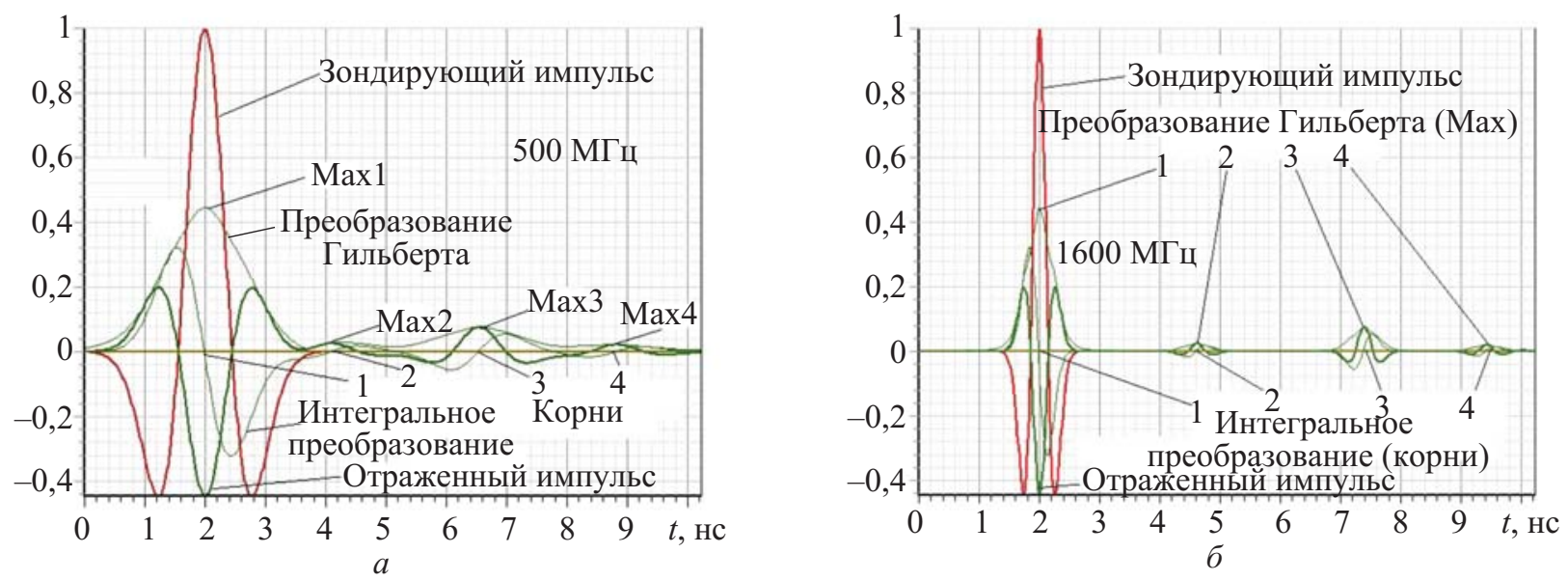

Рис. 6. Пример обработки сигнала георадара на частотах 500 (a) и 1600 МГц (б).

метров показаны на рис. 5 слева, где $H$ - геометрическая толщина соответствующего слоя в метрах.

Цель проведенных экспериментов состоит в том, чтобы найти возможность надежного определения моментов времени отраже- ния от внутренних границ. В соответствии с предлагаемой процедурой, для использования преобразования Гильберта после построения аналитического сигнала (2), на оси времени определяется расстояние между 


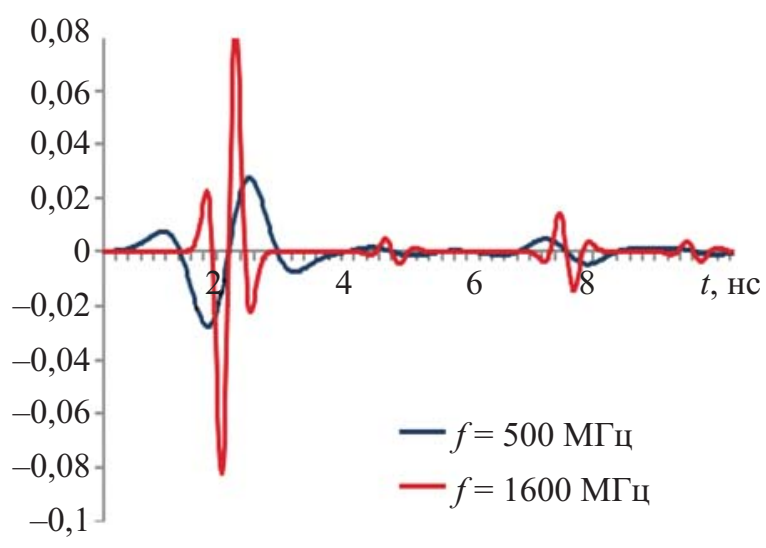

Рис. 7. Обработка производных от сигналов.

максимумами аналитического сигнала (рис. $6 a)$.

Очевидно, что с уменьшением толщины верхнего слоя, максимум отраженного сигнала и максимум преобразования Гильберта будут сдвигаться влево, и сливаться с первыми максимумами, соответствующими моменту отражения импульса от верхней границы и соответствующего преобразования Гильберта. Подобный график для сигнала с более высокой центральной частотой $f_{\mathrm{c}}$ показан на рис. $6 б$, где наблюдается более заостренная форма максимумов зависимости $S(t)$.

Однако амплитуды соответствующих максимумов на рис. 6 совпадают. Поэтому для увеличения надежности и удобства определения опорных точек сигналов в [20] предложено дополнительно вычислять производные функции, которая описывает интегральное преобразование. Полученный результат иллюстрируется на рис. 7. Такая функция обеспечивает более четкую индикацию опорных точек, вследствие более выраженного максимума. Это особенно важно в случае слабого отличия значений диэлектрической проницаемости слоев.

Наконец подчеркнем, что на глубину зондирования существуют известные физические ограничения, связанные с зависимостью ослабления сигнала от частоты. Поэтому отсутствует универсальное техническое решение, которое способно обеспечить и максимальную глубину зондирования, и высокую точность определения толщины слоев дорожного покрытия.

В этом смысле, для определения параметров верхних слоев структуры, которые имеют относительно малую толщину, когда важна точность, предпочтительно использовать РЛСПЗ с более высокой центральной частотой и предлагаемые методы обработки. Для изучения более глубоких слоев, имеющих значительную толщину, необходимо использовать РЛС с более низкой центральной частотой. Другим дополнительным результатом было подтверждение возможности виртуальной корректировки данных при использовании разработанного программного обеспечения GeoVizy.

К сожалению, взаимодействие сверхширокополосных сигналов, даже в рамках простейших моделей плоскослоистых сред, если принять во внимание реальные свойства конкретных реализаций антенн, является сложным явлением с точки зрения теории электромагнитного поля, и не может быть полностью описано с помощью простейших электродинамических моделей. Поэтому одним из предложений авторов этой статьи является последовательное использование различных антенных конфигураций для увеличения надежности получаемых оценок. В качестве примера в табли- 
Сравнительный анализ спектральных характеристик сигналов подповерхностных РЛС для оценки дорожного покрытия

Таблица 1. Наборы данных для георадаров с различными ичентральными частотами

\begin{tabular}{|c|c|c|c|c|c||}
\hline Георадар & $f_{c}$, МГц & Номер слоя & $\begin{array}{c}\text { Измеренная } \\
\text { толщина, см }\end{array}$ & $\begin{array}{c}\text { Реальная } \\
\text { толщина, см }\end{array}$ & $\begin{array}{c}\text { Относительная } \\
\text { ошибка, \% }\end{array}$ \\
\hline ТRF-3 & 500 & 1 & 11,5 & 12 & $-4,1$ \\
\hline Одяг-5 & 1050 & 1 & 12,4 & 12 & $+3,3$ \\
\hline Одяг-4 & 1600 & 1 & 11,9 & 12 & +1 \\
\hline ТRF-3 & 500 & 2 & 15,8 & 15 & $-4,6$ \\
\hline Одяг-5 & 1050 & 2 & 14,3 & 15 & $-3,3$ \\
\hline Одяг-4 & 1600 & 2 & 14,5 & 17 & $-3,5$ \\
\hline ТRF-3 & 500 & 3 & 16,4 & 17 & $-4,7$ \\
\hline Одяг-5 & 1050 & 3 & 16,2 & 17 & $+5,2$ \\
\hline Одяг-4 & 1600 & 3 & 17,9 & & +5 \\
\hline \hline
\end{tabular}

це 1 показаны результаты количественных оценок двух конструкций при использовании трех антенн с различными значениями центральной частоты.

Анализ полученных экспериментальных данных вновь подтверждает сложную природу волновых процессов, сопровождающих дифракцию электромагнитных волн в плоскослоистых средах. Полученные результаты подтверждают, что в настоящее время метод неразрушающего контроля обработки данных, предложенный авторами, является наиболее эффективным средством для оценки параметров плоскослоистых сред.

Очевидно, что с увеличением общей толщины рассматриваемой структуры, вследствие специфики схемы обработки [2], ошибка оценивания будет возрастать. Это обусловлено не только присутствием дисперсии, как отмечалось выше, но также эффектами рассеяния электромагнитных волн, вследствие шероховатости границ между слоями [31].

\section{7. ВЫВОДЫ}

Анализ особенностей различных антенных систем позволяет предложить эффективные модели для обработки и интерпретации данных зондирования плоскослоистых сред. В работе исследованы особенности сигнальных спектров различных антенных систем и варианты спектральной обработки СШП импульсных сигналов трех РЛСПЗ. Проведенные экспериментальные измерения сигналов на антенных блоках реальной РЛСПЗ с различными центральными частотами позволили установить возможности этих радаров подповерхностного зондирования для решения прикладных задач, прежде всего задач измерения толщины слоев дорожного покрытия.

Для увеличения надежности и точности определения значений толщины внутренних слоев плоскослоистых сред предложено дополнительное вычисление производных от функции, описывающей интегральное преобразование (рис. 7). Этот метод обработки сигнала показал хорошие результаты при использовании в процессе мониторинга дорожных покрытий. Кроме 
того, одновременное использование двух интегральных преобразований при обработке данных, а именно, преобразование Гильберта и преобразование, основанное на поиске корней обычного интеграла с переменным верхним пределом, также позволило увеличить надежность получаемых оценок для толщины слоев (табл. 1).

К сожалению, ограниченный объем статьи не позволяет охватить все вопросы, связанные с особенностями обработки импульс- ных сигналов. Надеемся, что заинтересованные читатели смогут ознакомиться с некоторыми результатами, полученными в работах [2, 17, 23, 26]. Кроме того, авторы планируют рассмотреть некоторые новые проблемные вопросы в будущих публикациях.

\section{БЛАГОДАРНОСТИ}

Эта работа поддержана Министерством образования и науки Украины в рамках гранта $0117 \mathrm{U} 004862$.

Поступила в редакцию 16.10.2020

После доработки 26.04.2021

Принята к публикации 28.04.2021

\section{IN F ORMATION ON THE ARTICLE}

\section{COMPARATIVE ANALYSIS OF SPECTRAL CHARACTERISTICS OF PULSE GPR SIGNALS FOR ROAD PAVEMENT ASSESSMENT}

Dmitriy O. Batrakov, ORCID: 000-0002-6726-8162, batrakov@karazin.ua, V. N. Karazin Kharkiv National University, Ukraine

Mariya S. Antyufeyeva, ORCID: 0000-0002-6654-4794, mariya.antyufeyeva@gmail.com, V. N. Karazin Kharkiv National University, Ukraine

Angelika G. Batrakova, ORCID: 0000-0002-4067-4371, agbatr@ukr.net, Kharkiv National Automobile and Highway University, Ukraine

Results of experiments on the use of three antenna units of ultra-wideband ground penetrating radars (UWB GPR) for nondestructive testing are presented. These three units have both different values of the centres frequency of the probing UWB pulses and different antenna configurations. The purpose of the performed experiments was to find the best values of sensing parameters. Another task was to optimize the technique for processing signals reflected from plane-layered media. The performed analysis of the obtained results showed the ways for improving the quality of nondestructive testing of road pavement and other building structures by using the UWB GPR. The proposed approach is based on the previously offered algorithm for the stepwise determination of the pavement layer thickness and the relative dielectric permittivity.

Keywords: non-destructive testing; UWB signals; GPR; road pavements

\section{REFERENCES}

1. H. Jol, Ed., Ground Penetrating Radar Theory and Applications. Amsterdam: Elsevier B.V., 2008, uri: https://www.elsevier.com/books/ground-penetrating-radar-theor y-and-applications/jol/978-0-444-53348-7.

2. G. P. Pochanin, V. P. Ruban, P. V. Kholod, A. A. Shuba, A. G. Pochanin, A. A. Orlenko, "Enlarging of power budget of ultrawideband radar," in 2013 6th International Conference on Recent Advances in Space Technologies (RAST), 2013, pp. 213-216, doi: 10.1109/RAST.2013.6581203.

3. D. O. Batrakov, N. P. Zhuck, "Solution of a general inverse scattering problem using the distorted Born approximation and iterative technique," Inverse Probl., vol. 10, no. 1, pp. 39-54, 1994, doi: 10.1088/0266-5611/10/1/004.
4. T. Saarenketo, "Electrical properties of road materials and subgrade soils and the use of ground penetrating radar in traffic infrastructure surveys," 2006. uri: http://jultika.oulu.fi/ files/isbn9514282221.pdf.

5. S. Pengcheng, "Development of algorithms for asphalt pavement compaction monitoring utilizing ground penetrating radar," Urbana, 2014. uri: https://core.ac.uk/download/pdf/291 75239.pdf.

6. "Smart Pavement Monitoring System. Report No. FHWA-HRT-12-072," 2013. uri: https://www.fhwa.dot.gov/ publications/research/infrastructure/pavements/12072/12072.pd f.

7. G. P. Arnold, F. Sing, T. Saarenketo, T. Saarenpaa, "Pavement moisture measurement to indicate risk to pavement life," NZ Transp. Agency Res., no. Report 611, 2017, uri: https://www.nzta.govt.nz/resources/research/reports/611/. 
8. Z. Dong et al., "Rapid detection methods for asphalt pavement thicknesses and defects by a vehicle-mounted ground penetrating radar (GPR) system," Sensors, vol. 16, no. 12, p. 2067, 2016, doi: 10.3390/s16122067.

9. J. Hu, P. K. R. Vennapusa, D. J. White, I. Beresnev, "Pavement thickness and stabilised foundation layer assessment using ground-coupled GPR," Nondestruct. Test. Eval., vol. 31, no. 3, pp. 267-287, 2016, doi: 10.1080/10589759.2015.1111890.

10. L. Krysinski, J. Sudyka, "Typology of reflections in the assessment of the interlayer bonding condition of the bituminous pavement by the use of an impulse high-frequency ground-penetrating radar," Nondestruct. Test. Eval., vol. 27, no. 3, pp. 219-227, 2012, doi: 10.1080/10589759.2012.674525.

11. J. Sudyka, L. Krysinski, "Evaluation of homogeneity of thickness of new asphalt layers using GPR," IOP Conf. Ser. Mater. Sci. Eng., vol. 356, no. 1, p. 012025, 2018, doi: 10.1088/1757-899X/356/1/012025.

12. T. Xia, D. Huston, "High speed ground penetrating radar for road pavement and bridge structural inspection and maintenance," 2016. uri: https://www.uvm.edu/sites/default/ files/Transportation-Research-Center/Reports/2020 and more/2016 _ _ High_Speed_Ground_Penetrating_Radar_for_Ro ad Pavement and Bridge Structural Inspection and Mainten ance.pdf? $\mathrm{t}=\mathrm{qk} 1 \mathrm{rlm}$.

13. D. O. Batrakov, M. S. Antyufeyeva, A. V. Antyufeyev, A. G. Batrakova, "Remote sensing of plane-layered media with losses using UWB signals," in 2017 XI International Conference on Antenna Theory and Techniques (ICATT), 2017, pp. 370-373, doi: 10.1109/ICATT.2017.7972666.

14. A. De Coster, A. Van der Wielen, C. Gregoire, S. Lambot, "Evaluation of pavement layer thicknesses using GPR: A comparison between full-wave inversion and the straight-ray method," Constr. Build. Mater., vol. 168, pp. 91-104, 2018, doi: 10.1016/j.conbuildmat.2018.02.100.

15. P. Eskelinen, T. Pellinen, "Comparison of different radar technologies and frequencies for road pavement evaluation," Constr. Build. Mater., vol. 164, pp. 888-898, 2018, doi: 10.1016/j.conbuildmat.2018.01.124.

16. D. O. Batrakov, M. S. Antyufeyeva, A. V. Antyufeyev, A. G. Batrakova, "UWB signal processing during thin layers thickness assessment," in 2016 IEEE Radar Methods and Systems Workshop (RMSW), 2016, pp. 36-39, doi: 10.1109/RMSW. 2016.7778545.

17. R. A. Tarefder, M. U. Ahmed, "Ground penetrating radar for measuring thickness of an unbound layer of a pavement," in Advances in Intelligent Systems and Computing, vol. 598, 2018, pp. $160-167$.

18. D. Batrakov, A. Batrakova, M. Antyufeyeva, "Combined GPR data analysis technique for diagnostics of structures with thin near-surface layers," Diagnostyka, vol. 19, no. 3, pp. 11-20, 2018, doi: 10.29354/diag/91489.

19. D. O. Batrakov, M. S. Antyufeyeva, A. V. Antyufeyev, A. G. Batrakova, "Inverse problems and UWB signals in biomedical engineering and remote sensing," in 2016 8th International Conference on Ultrawideband and Ultrashort
Impulse Signals (UWBUSIS), 2016, pp. 148-151, doi: 10.1109/ UWBUSIS.2016.7724174

20. D. O. Batrakov, M. S. Antyufeyeva, A. V. Antyufeyev, A. G. Batrakova, "GPR data processing for evaluation of the subsurface cracks in road pavements," in 2017 9th International Workshop on Advanced Ground Penetrating Radar (IWAGPR), 2017, pp. 1-6, doi: 10.1109/IWAGPR.2017.7996072.

21. D. O. Batrakov, M. S. Antyufeyeva, A. G. Batrakova, V. V. Troyanovsky, M. O. Pilicheva, "UWB signal processing for the road pavements assessment," in 2019 IEEE 2nd Ukraine Conference on Electrical and Computer Engineering (UKRCON), 2019, pp. 192-195, doi: 10.1109/UKRCON.2019. 8879866.

22. T. Uno, S. Adachi, "Inverse scattering method for one-dimensional inhomogeneous layered media," IEEE Trans. Antennas Propag., vol. 35, no. 12, pp. 1456-1466, 1987, doi: 10.1109/TAP.1987.1144033.

23. A. G. Tijhuis, C. van der Worm, "Iterative approach to the frequency-domain solution of the inverse-scattering problem for an inhomogeneous lossless dielectric slab," IEEE Trans. Antennas Propag., vol. 32, no. 7, pp. 711-716, 1984, doi: 10.1109/TAP.1984.1143410.

24. D. O. Batrakov, "Quality and efficiency of data analysis in multifrequency radio-wave testing of laminated dielectrics," Russ. J. Nondestruct. Test., vol. 34, no. 8, pp. 612-618, 1998.

25. T. M. Habashy, W. C. Chew, E. Y. Chow, "Simultaneous reconstruction of permittivity and conductivity profiles in a radially inhomogeneous slab," Radio Sci., vol. 21, no. 4, pp. 635-645, 1986, doi: 10.1029/RS021i004p00635.

26. S. He, "Frequency and time domain Green function technique for nonuniform LCRG transmission lines with frequency-dependent parameters," J. Electromagn. Waves Appl., vol. 7, no. 1, pp. 31-48, 1993, doi: 10.1163/156939393X01065.

27. D. O. Batrakov, N. P. Zhuk, "Method for testing of layer-non-homogeneous dielectrics using numerical solution of reverse problem dialing with dissipation in polarization parameters domain," Defektoskopiya, no. 2, pp. 82-87, 1994.

28. S. Zhao, P. Shangguan, I. L. Al-Qadi, "Application of regularized deconvolution technique for predicting pavement thin layer thicknesses from ground penetrating radar data," NDT E Int., vol. 73, pp. 1-7, 2015, doi: 10.1016/j.ndteint.2015.03.001.

29. L. Y. Astanin, A. A. Kostylev, Ultrawideband Radar Measurements: Analysis and Processing. The Institution of Engineering and Technology, Michael Faraday House, Six Hills Way, Stevenage SG1 2AY, UK: IET, 1997, doi: 10.1049/ PBRA007E.

30. L. Y. Astanin, M. V. Kipke, V. V. Kostyleva, "The structural features of ultrawideband signals," in 2008 th International Conference on Ultrawideband and Ultrashort Impulse Signals, 2008, pp. 180-182, doi: 10.1109/UWBUS. 2008.4669402.

31.D. Batrakov, A. Batrakova, S. Urdzik, R. Danielyan, "Nondestructive diagnostics and detection of subsurface cracks in non-rigid pavements with GPR," Diagnostyka, vol. 22, no. 2 , pp. 85-95, 2021, doi: 10.29354/diag/137915. 